\title{
EL GASTO REGIONAL EN LA DINAMICA CULTURAL ESPAÑOLA
}

\author{
POR \\ Pedro Plasencia
}

SUMARIO: Introducción.-1. EI marco de la acción cultural de las Comunidades Autónomas y sus instrumentos financieros. - II. La actividad de gasto de las Comunidades Autónomas y del Estado; principales tendencias y configuración de políticas culturales propias.-III. Las televisiones autonómicas en la dinámica cultural.-IV. Conclusiones.

Fuentes: Presupuestos Generales del Estado 1984-1987, Presupuestos de las Comunidades Autónomas 1985-1987, Programa de Inversiones Públicas 1985-1987 y Financiación de Comunidades Autónomas, INAP, marzo 1987.

\section{INTRODUCCION}

El proceso que a partir de 1976 lleva a una descentralización política del Estado español con la instauración de la democracia, ha corrido paralelo a una dinamización cultural creciente del total de la sociedad española, como lo demuestran tanto el incremento del gasto público en el sector cultural como la emergencia de nuevas instancias y figuras de financiación privada.

Una refexión sobre hasta qué punto los flujos democratizacióndinamización cultural, regionalización-dinamización cultural, vienen siendo interdependientes $y$, en consecuencia, sobre cuál ha sido y es el papel de las Comunidades Autónomas en la extensión de los fenómenos culturales y en la definición de políticas culturales, es el propósito de este artículo.

Para ello, partiendo del marco que para la acción cultural de las Comunidades Autónomas (CC. AA.) fijan la Constitución y los Estatutos de Autonomía, después de hacer un breve repaso de la evolución de los instrumentos reales y financieros con los que los Entes regionales han contado para gestionar los servicios culturales asumidos en el referido marco competencial y diseñar una política cultural propia, nos detendremos en la actividad de gasto de las CC. AA. y del Estado y sus Organismos Autónomos (OO. AA.) culturales para estudiar el comportamiento y las tendencias más significativas. Dentro de la misma perspectiva del gasto intentaremos recoger unas notas aisladas sobre la diversificación del mismo 
por programas o sectores culturales y su eficiencia, terminando por exponer alĝunas conclusiones a las que nos llevará el análisis de los puntos anteriores.

\section{i. EL MARCO DE LA ACCION CULTURAL DE LAS COMUNIDADES AUTONOMAS Y SUS INSTRUMENTOS FINANCIEROS}

El marco de la actividad cultural de las Comunidades Autónomas, diseñado por la Constitución Española de 1978, en sus artículos 148 y 149, y completado por los sucesivos Estatutos de Autonomía que fueron promulgándose en los años siguientes, se ha ido colmando a medida que las entidades regionales han ido contando con los instrumentos reales y financieros necesarios para ello.

De acuerdo con el texto constitucional, las Comunidades Autónomas pueden asumir competencias en museos, bibliotecas, conservatorios de música y patrimonio monumental de interés de la Comunidad Autónoma; fomento de la cultura, de la investigación y, en su caso, de la enseñanza de la lengua de la Comunidad Autónoma, así como promoción del deporte y la adecuada utilización del ocio. El Estado, por su parte, tiene competencia exclusiva para dictar las normas básicas del régimen de prensa, radio y televisión $y$, en general, de todos los medios de comunicación social, $\sin$ perjuicio de las facultades que en su desarrollo $y$ ejecución correspondan a las Comunidades Autónomas, así como en la defensa del patrimonio cultural artístico y monumental español contra la exportación y la expoliación; museos, bibliotecas y archivos de titularidad estatal, sin perjuicio de su gestión por parte de las Comunidades Autónomas.

Las materias no atribuidas expresamente al Estado por la Constitución podrían corresponder a las Comunidades Autónomas, en virtud de sus respectivos Estatutos.

En cuanto a la instrumentación del campo competencial cabe distinguir dos fases que vienen separadas por la promulgación de la Ley Orgánica de Financiación de las Comunidades Autónomas de 22 de septiembre de 1980 (LOFCA).

Antes de la publicación de la LOFCA, las Comunidades Autónomas $y$ entes preautonómicos vinieron recibiendo una serie de transferencias de servicios y medios personales, que se efectuaron por Decreto, y de transferencias financieras directas de los Presupuestos Generales del Estado. 
La LOFCA, por su parte, establece los siguientes instrumentos de financiación: a) los ingresos procedentes del patrimonio propio y demás de derecho privado; b) los propios impuestos, tasas y contribuciones especiales; c) los tributos cedidos parcial o totalmente por el Estado; d) recargos sobre los impuestos del Estado; e) participaciones en los ingresos del Estado; f) el producto de las operaciones de crédito; gl producto derivado de la potestad sancionadora; $h$ ) asignaciones establecidas en los Presupuestos Generales del Estado; i) transferencias del Fondo de Cooperación Interterritorial.

A estos recursos, en 1987 se han añadido: a) Las compensaciones reconocidas a las Comunidades Autónomas para la liquidación del período transitorio (pagos por una sola vez). b) Los fondos provenientes del FEDER.

La financiación prevista en la LOFCA, salvo para las Comunidades que gozan de Concierto o Convenio Económicos (País Vasco y Navarra), se ha hecho en dos fases. En la primera se ha aplicado un sistema transitorio recientemente liquidado, de cupos rígidos de participación en los ingresos del Estado y compensaciones de diversa índole. Concluido el período de transferencias y evaluado el coste efectivo de los servicios transferidos, se ha dado fin al período transitorio y los instrumentos LOFCA han cobrado toda su virtualidad para servir a una auténtica autonomía financiera que permita la definición de políticas culturales propias, así como de las demás políticas sectoriales.

En la experiencia recientemente adquirida se ha podido valorar el. papel mayor o menor que pueden jugar los diferentes instrumentos LOFCA; demostradas la escasa capacidad fiscal-tributaria propia de la Región (salvo para las tasas), la dificultad extrema para hacer efectivos los recargos sobre impuestos estatales, y las limitaciones del Fondo de Cooperación Interterritorial, concebido como subvención a la inversión condicionada para determinados proyectos, los principales recursos se han demostrado las asignaciones presupuestarias por participación en ingresos del Estado de tipo flexible (negociable y revisable) y las tasas y tributos cedidos por el Estado. Los recursos disponibles por parte de las Comunidades Autónomas por estos dos conceptos medidos en millones de pesetas, han sido los que siguen:

\begin{tabular}{l|c|c}
\hline & 1986 & 1987 \\
\cline { 2 - 3 } \multicolumn{1}{c|}{ Participación en ingresos del Estado } & 422.881 & 651.656 \\
Tasas y tributos cedidos ........... & 211.404 & 221.945 \\
\hline
\end{tabular}


El Fondo de Cooperación Interterritorial, que ha bajado de 209.000 millones de pesetas en 1984 hasta 141,200 en 1987, es el tercer recurso en importancia.

Por lo que se refiere a la parte de las inversiones públicas en cultura han detraído del Fondo de Cooperación Interterritorial en los últimos años; el siguiente cuadro demuestra la recesión de las mismas.

\section{INVERSIONES CULTURALES DEL FONDO DE COOPERACION INTERTERRITORIAL EN MILLONES DE PESETAS}

\begin{tabular}{c|c|c|c}
\hline 1984 & 1985 & 1986 & 1987 \\
\hline 5.124 & 6.185 & 6.058 & 2.898 \\
\hline
\end{tabular}

II. LA ACTIVIDAD DE GASTO DE LAS COMUNIDADES AUTONOMAS Y DEL ESTADO; PRINCIPALES TENDENCIAS Y CONFIGURACION DE POLITICAS CULTURALES PROPIAS

Las suficientes dotaciones de recursos y la capacidad de autonomía financiera condicionan la existencia de una política cultural regional, pero es la actividad de gasto la que configura las opciones, prioridades, objetivos, etc., que se constituyen en definitiva en los elementos de una política cultural propia.

Para saber cuáles están siendo estos objetivos y prioridades, así como los mecanismos utilizados para su prosecución en las diferentes Comunidades Autónomas, hemos analizado los Presupuestos por Programas de éstas, de cuyo análisis se ha podido evidenciar las tendencias comunes a todas las regiones y las divergencias en cuanto a política de gasto, así como comparar los comportamientos regionales con los del Estado y sus Organismos Autónomos administrativos con competencias en cultura.

Las principales tendencias observadas han sido las siguientes:

1. Incremento más dinámico del gasto en actividades culturales por parte de las Comunidades Autónomas que por parte del Estado

El mayor dinamismo en el gasto por parte de las Comunidades Autónomas se evidencia tanto en la evolución de los créditos para programas culturales como en la participación creciente en el montante de inversiones públicas en cultura y deporte. 
Por lo que se refiere al total de créditos para programas culturales, mientras que los consignados para el Ministerio de Cultura y sus Organismos Autónomos han sufrido un estancamiento $e$, incluso, un retroceso de 1985 a 1986, como lo demuestran las cifras que siguen:

Presupuesto del Estado para Cultura en millones de pesetas:

1984: 49.933.

1985: 52.898.

1986: 50.087.

1987: 51.892

El conjunto de los créditos asignados a los programas de cultura y deporte de las Comunidades Autónomas ha pasado de una cantidad mínima en 1983 a una cifra que ronda los 55.000 millones de pesetas en el presente ejercicio presupuestario en el que ya ha superado el total del gasto cultural en manos de la Administración Central del Estado y sus Organismo Autónomos.

En cuanto a la participación de las Comunidades Autónomas en el montante de las inversiones públicas en cultura y deporte, de 3.825 millones de pesetas en 1984 , lo que representa un 19,4 por 100 sobre el total de las inversiones públicas, que ascendió ese año a 19.667 millones de pesetas, se ha pasado en 1987 a una cifra de 11.350 millones de pesetas, que representa un 43 por 100 sobre un total de 26.277 millones de pesetas.

Indudablemente esta dinámica de incremento ha sido motivada principalmente por el proceso de transferencias de competencias del Estado que se ha llevado a cabo a un ritmo altamente satisfactorio; sin embargo, y aunque hoy pueda darse por concluido este proceso de transferencias, no sería extraño que en los próximos años continuara la tendencia expansiva en la actividad de gasto cultural de las Comunidades Autónomas o, al menos, de algunas de ellas, y ello por diferentes razones:

a) Elmayor potencial financiero de las Comunidades Autónomas en 1987, debido a la liquidación del período transitorio que ha permitido un incremento neto de financiación de 60.000 millones de pesetas de 1986 a 1987, y a otros factores más duraderos, no dejará de producir en los próximos años el «efecto demostración» característico del gasto público.

b) Como consecuencia de la entrada en vigor del sistema definitivo de financiación se produce un notable incremento de la financiación incondicional, esto es, de aquella financiación que las Comunidades Autónomas reciben del Estado sin que exista ningún 
tipo de condicionamiento a cómo debe ser empleada, una vez garantizado el nivel de atención a los servicios que las Comunidades Autónomas deben prestar como consecuencia de las competencias que éstas han ido adquiriendo del Estado.

c) La posibilidad de contar con un modelo de financiación a la carta, dada la diversidad de instrumentos, permite nuevas posibilidades de gasto en función de la mayor capacidad de esfuerzo fiscal de una Comunidad Autónoma.

Estos factores llevan conjuntamente a las Comunidades Autónomas a poder definir desde una mayor autonomía financiera su propia política cultural en el futuro.

\section{Mayor productividad del gasto cultural asumido por las Comunidades Autónomas que el del Estado}

Mucho más interesante que el estudio de las tendencias en la evolución de las cantidades del gasto público regional y estatal en cultura resulta el análisis de la calidad de ese gasto, medida en los términos de la relación entre gastos corrientes, con respecto a gastos de inversión y de gastos consuntivos con respecto a inversión más transferencias corrientes.

En este sentido, y aunque se comprueba una gran diversidad de comportamientos entre las distintas Comunidades Autónomas, puede afirmarse que los servicios asumidos por éstas presentan con respecto a los que restan en manos del. Estado una mayor productividad.

Se ofrece a continuación un cuadro que viene a recoger los porcentajes de los gastos de inversión, entendiendo por tales los capítulos VI y VII de los Presupuestos, por un lado, y, por otro, de los gastos de inversión más las transferencias corrientes (cap. IV). sobre el total de los créditos presupuestados en 1987 referidos a once Comunidades Autónomas y al Estado y sus Organismos Autónomos, haciendo notar que en las Comunidades Autónomas marcadas con un * el porcentaje ha sido hallado incluyendo los programas de Juventud y Deporte y en las demás únicamente los genuinamente culturales.

Como se desprende de la comparación de cifras, nueve de las once Comunidades Autónomas superan al Estado si nos acogemos al criterio de la inversión, y el total de ellas si añadimos las transferencias corrientes.

En cuanto a las medidas de las Comunidades Autónomas consideradas, éstas superan en nueve puntos porcentuales $y$ en 15,8, respectivamente, la cifra del Estado. 
No obstante, cabe hacer las siguientes observaciones:

- Las cifras dadas tienen una significación solamente relativa dada la evidente productividad social de algunos conceptos presupuestarios dentro del capítulo II y que no son considerados como gastos productivos en este estudio.

- Al considerar un solo año presupuestario y no una media ponderada de varios o la evolución de un período determinado, algunas Comunidades Autónomas pueden figurar con una cifra muy baja o muy alta de inversión que puede ser debida a circunstancias coyunturales.

- La diferencia entre los porcentajes de gasto recogidos refleja una mayor productividad del gasto regional que sin duda se debe parcialmente a la mayor abundancia en competencias de gestión por parte de las regiones frente a las competencias en planificación, ordenación general, etc., por parte del Estado.

CUADRO I

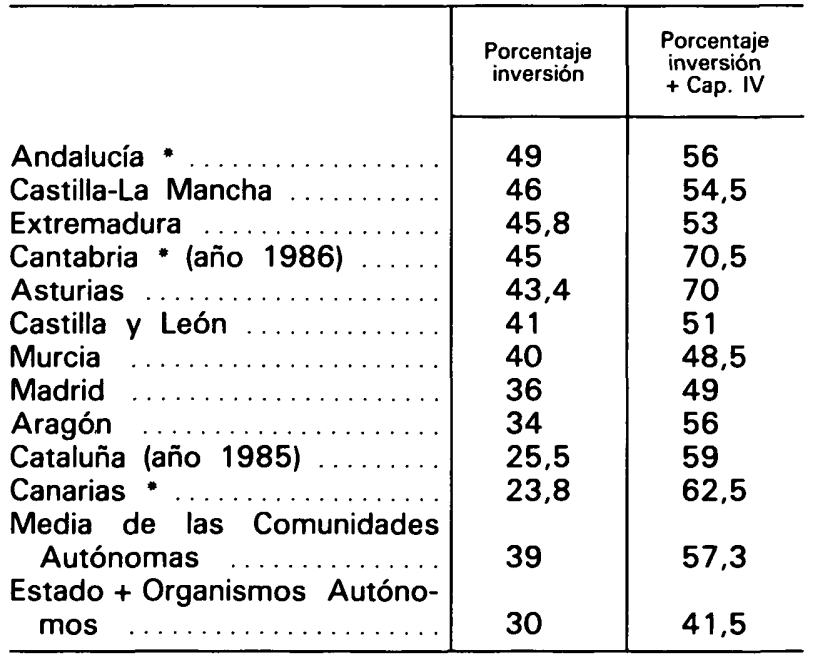

\section{Gran diversidad de las politicas culturales desde el punto de vista del gasto}

La importancia que a los distintos programas culturales le han dado las diferentes Comunidades Autónomas varía mucho de unas a otras. En algunos casos la existencia de programas para la protección y el fomento de una cultura autóctona derivada del hecho lingüístico justifica un mayor esfuerzo en los programas 
culturales; en otros, el hecho motivador de una política cultural puede ser la existencia de un patrimonio histórico artístico muy vasto y necesitado de conservación y restauración, como sucede con las Comunidades de Castilla y León, y Andalucía; en otros, finalmente, el motor del gasto ha sido la fuente de inversión pública en infraestructuras culturales.

Como quiera que sea, los porcentajes de los gastos en programas culturales sobre el total de los Presupuestos regionales varía mucho de una Comunidad a otra. Teniendo en cuenta el hecho de que algunas Comunidades Autónomas disponen de unos presupuestos más cuantiosos por haber asumido competencias de Educación y en otros sectores, y que los gastos culturales pueden ser presentados incluyendo o no los programas de juventud $y$ deporte, el siguiente cuadro puede ilustrar la diversidad a la que hacemos referencia con relación a algunas Comunidades Autónomas.

CUADRO ॥

CC. AA. CON COMPETENCIAS EN EDUCACION

\begin{tabular}{|c|c|c|c|}
\hline Comunidades & Cultura & $\begin{array}{l}\text { Cultura, } \\
\text { Juventud } \\
\text { y Deporte }\end{array}$ & $\begin{array}{c}\text { Cultura } \\
\text { y Deporte }\end{array}$ \\
\hline $\begin{array}{l}\text { Andalucía } \ldots \ldots \ldots \ldots \ldots \\
\text { Canarias } \ldots \ldots \ldots \ldots \ldots \\
\text { Galicia } \ldots \ldots \ldots \ldots \ldots \\
\text { País Vasco (año 1986). }\end{array}$ & $\begin{array}{l}- \\
\overline{1}, \overline{55} \\
2,11\end{array}$ & $\begin{array}{l}1,76 \\
2,77 \\
- \\
-\end{array}$ & $\begin{array}{l}- \\
- \\
-\end{array}$ \\
\hline
\end{tabular}

CC. AA. SIN COMPETENCIAS EN EDUCACION

\begin{tabular}{|c|c|c|c|}
\hline Comunidades & Cultura & $\begin{array}{c}\text { Cultura, } \\
\text { Juventud } \\
\text { y Deporte }\end{array}$ & $\begin{array}{c}\text { Cultura } \\
y \text { Deporte }\end{array}$ \\
\hline $\begin{array}{l}\text { Asturias } \ldots \ldots \ldots \\
\text { Cantabria (año 1986) } \\
\text { Castilla y León } \ldots \ldots \ldots \\
\text { Extremadura } \ldots \ldots \ldots \\
\text { Madrid } \ldots \ldots \ldots \ldots \ldots \\
\text { Murcia } \ldots \ldots \ldots \ldots \\
\text { Estado } \ldots \text { Organismos } \\
\text { Autónomos } \ldots \ldots \ldots\end{array}$ & $\begin{array}{l}2,82 \\
5 \\
4,4 \\
3,2 \\
1,78 \\
3,16 \\
0,38\end{array}$ & $\begin{array}{l}5,38 \\
- \\
- \\
- \\
- \\
6,40 \\
0,58\end{array}$ & $\begin{array}{c}-\overline{7} \\
- \\
5,46 \\
3,55 \\
-\end{array}$ \\
\hline
\end{tabular}




\section{LAS TELEVISIONES AUTONOMICAS EN LA DINAMICA CULTURAL}

Habida cuenta de la función de cohesión que la comunicación tiene sobre el tejido social, una mención muy especial cabe hacer del gran apoyo que para la configuración de políticas culturales propias ha supuesto el nacimiento de una televisión autónoma en las Comunidades históricas del Estado español (Galicia, País Vasco y Cataluña).

Las televisiones autonómicas contribuyen en una forma, de la que evidentemente los canales regionales de la televisión estatal están muy lejos, a la formación de una identidad regional que es la base de la identidad cultural y de la autonomía política, convirtiéndose en uno de los elementos más dinámicos de la vida cultural regional como soporte de actividades culturales tanto como fenómeno cultural en sí mismo.

Reflejamos simplemente el gasto presupuestado en millones de pesetas para los distintos entes y sociedades gestoras de radiodifusión y televisión en el ejercicio de 1986 en las tres Comunidades Autónomas con televisión autonómica, sin extendernos en mayores consideraciones.

\section{País Vasco}

Ente público R.T.V.V.: 3.288.

Presupuesto consolidado: 4.564 .

\section{Galicia}

Sociedad Gestora del Servicio Público Radiodifusión: 300.

Sociedad Gestora del Servicio Público de Televisión: 2.805.

\section{Cataluña}

Televisión de Cataluña, S. A.: 8.389.

Cataluña Radio Servicio: 877.

\section{CONCLUSIONES}

Con ánimo de síntesis, algunas pequeñas conclusiones pueden ser extraídas de lo expuesto; nos aventuramos a señalar que:

1. A la descentralización del sector servicios culturales, consecuencia de la descentralización política obrada por el nuevo 
sistema constitucional, ha venido ligada una expansión de las actividades culturales; con ello han podido demostrarse dos premisas ya conocidas: a) que la democratización de una sociedad contribuye a la eclosión de sus culturas y subculturas, y b) que la instancia regional es. la instancia ideal para la definición de políticas culturales autónomas, la dinamización de los diferentes agentes culturales y la gestión de los servicios culturales.

2. El reparto de competencias que llevan a cabo la Constitución y los Estatutos de Autonomía permiten a las Comunidades Autónomas el diseño de sus propias políticas culturales; éste, sin embargo, se ha visto dificultado por el sistema de financiación vigente prácticamente hasta el ejercicio pasado. En la actualidad las Comunidades Autónomas cuentan con los instrumentos necesarios para, de una forma operativa, fijar los objetivos, prioridades $y$ recursos que son los elementos de una autodefinición cultural.

3. Partiendo de una relativa igualdad en cuanto a las posibilidades a priori, las regiones y nacionalidades que componen el Estado español han actuado en materia cultural de muy diversa manera dedicando mayores o menores esfuerzos económicos a la cultura, dando prioridades a sectores culturales y programas diversos, etc. Las Comunidades históricas, al contar con la riqueza de una cultura autóctona en lengua propia distinta al español y con el instrumento de unos medios de comunicación propios y autonómicos, han empleado más recursos y han podido incidir con mayor operatividad sobre la defensa de la identidad cultural; ello, no obstante, es digno de elogio el esfuerzo realizado por otras regiones como Andalucía, que se sitúa a la cabeza con gran diferencia en el esfuerzo económico cultural, o como Cantabria, con el porcentaje más alto de gasto cultural. 
REALA-1989, núm. 244. PLASENCIA, PEDRO. EL GASTO REGIONAL EN LA DINAMICA CULTURA...

REALA-1989, núm. 244. PLASENCIA, PEDRO. EL GASTO REGIONAL EN LA DINAMICA CULTUURA... 